\section{ZEW}

Zentrum für Europäische Wirtschaftsforschung GmbH

Centre for European Economic Research

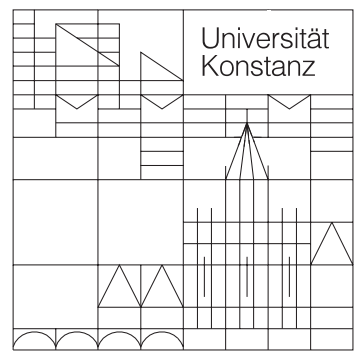

Rechts-, Wirtschafts- und Verwaltungswissenschaftliche Sektion

Fachbereich

Wirtschaftswissenschaften

Diskussionspapiere der DFG-

Forschergruppe (Nr.: 3468269275):

Heterogene Arbeit: Positive und Normative Aspekte der Qualifikationsstruktur der Arbeit

Heinrich W. Ursprung

Schneewittchen im Land der

Klapperschlangen: Evaluation eines Evaluators

Dezember 2002 


\title{
Schneewittchen im Land der Klapperschlangen: Evaluation eines Evaluators
}

\author{
Heinrich W. Ursprung
}

\author{
Universität Konstanz \\ Fach D138 \\ 78457 Konstanz \\ Germany \\ mail : heinrich.ursprung@uni-konstanz.de \\ phone : +49-7531-88-2332 \\ fax : $+49-7531-88-3130$
}

\section{Zusammenfassung:}

Dieses Papier kritisiert das kürzlich vom halboffiziellen "Centrum für Hochschulentwicklung" (CHE) veröffentlichte Forschungsranking für die deutschen volkswirtschaftlichen Fachbereiche. Es wird gezeigt, dass die Vernachlässigung vernünftiger Qualitätsindikatoren zu einem Ranking führt, das für eine forschungspolitische Standortbestimmung unbrauchbar ist. Die vom CHE verwendete Methode der Messung des Forschungsoutputs würde darüber hinaus als Führungsinstrument der Universitätsleitung geradezu kontraproduktiv wirken, da sie nicht anreizkompatibel ist.

\begin{abstract}
:
This is a comment on a ranking of German economics departments published by a semi-official institution, the Center for Higher Education Development (CHE). It is shown that the $\mathrm{CHE}$ ranking is highly misleading because the publication output is not weighted according to quality. The CHE ranking is compared to the results of four recent ranking studies commissioned by the European Economic Association (EEA) and an earlier ranking of German, Austrian and Swiss economics departments. For the readers from Austria and Switzerland we include in the appendix a "peek preview" of the "EEA rankings" which includes the top-ranked Austrian and Swiss departments.
\end{abstract}

\author{
JEL Classification : A14, I29 \\ Keywords : : ranking economics departments \\ Download/Reference : http://www.ub.uni-konstanz.de/kops/volltexte/2003/1037
}




\title{
Schneewittchen im Land der Klapperschlangen: Evaluation eines Evaluators
}

\author{
Heinrich W. Ursprung* \\ Fachbereich Wirtschaftswissenschaften \\ Universität Konstanz \\ Fach D-138 \\ D-78457 Konstanz
}

erscheint in den Perspektiven der Wirtschaftspolitik (Nr. 2, 2003)

Vor fünf Jahren haben Rolf Bommer und ich der volkswirtschaftlichen Profession den Spiegel vorgehalten, um die forschungsstärksten Fachbereiche in Deutschland, Österreich und der Schweiz zu bestimmen (,Spieglein, Spieglein and der Wand“, ZWS, 1998). Unser Forschungsranking - erschienen in der Vorgängerin dieser Zeitschrift -- wollten wir damals insbesondere als Aufforderung verstanden wissen, in Zukunft die universitären Forschungsleistungen professionell und regelmäßig quantitativ erfassen zu lassen. Auch wenn es so scheinen mag, Ranglisten dienen eben nicht bloß eitlen Wissenschaftlern zum Zeitvertreib: professionell erstellte Forschungsrankings sind aus zweierlei Gründen von hochschulpolitischer Bedeutung. Erstens muss jede rationale Hochschulpolitik in der Lage sein, ihre Schneewittchen -- die in voller Blüte stehenden und die knospenden -- eindeutig zu identifizieren. Nur so kann die Forschung effizient gefördert werden. Zweitens ist die in Forschungsrankings enthaltene Information aber auch für die betroffenen Wissenschaftler und für den wissenschaftlichen Nachwuchs von Bedeutung, wenn es darum geht, einen geeigneten Standort für die eigene Forschung bzw. Weiterbildung zu finden. Qualitativ hochstehende Forschungsrankings tragen maßgeblich zur Markttransparenz bei und erhöhen so die Effizienz der Forschung.

\footnotetext{
* Ich danke Tom Coupé, Laurent Linnemer, Michel Lubrano und Thanasis Stengos für zusätzliche Informationen über ihre Rankingstudien, und Laszlo Goerke, Mathias Kifmann, Eric Lehmann und Stanislav Nastassine für wertvolle Kommentare. Dank gebührt aber auch dem Herausgeber der $P W P$, Friedrich Schneider, für die äußerst effiziente Abwicklung des Publikationsprozesses.
} 
In der Zwischenzeit ist vom Centrum für Hochschulentwicklung (CHE) der Anspruch erhoben worden, aussagekräftige Forschungsrankings deutscher Universitäten für 11 verschiedene wissenschaftliche Disziplinen anbieten zu können. Unter anderem hat das CHE im November letzten Jahres ein Forschungsranking für das Fach Volkswirtschaftslehre publiziert (Berghoff et al., 2002). Die von uns vor fünf Jahren verlangte Professionalisierung im Rankingbusiness ist damit aber leider nicht erfolgt. Das CHE Forschungsranking für das Fach VWL ist nämlich weit davon entfernt, die Ansprüche, die man heutzutage an eine derartige Studie stellen muss, zu erfüllen.

Die CHE Evaluatoren sind offenbar mit der umfangreichen einschlägigen Literatur nicht genügend vertraut und legen ein Ranking vor, das höchstens dem methodologischen Entwicklungsstand der frühen 1980er Jahre entspricht. Das vorgelegte Ranking vermittelt denn auch einen völlig falschen rückblickenden Eindruck von der deutschen Forschungslandschaft im Bereich der VWL und ist somit als Instrument der Standortbestimmung unbrauchbar. Ebenso unbrauchbar ist das CHE Forschungsranking aber auch als Instrument der vorausschauenden Vorgabe von Forschungszielen und einer damit eventuell verbundenen Belohnung von Forschungsleistungen; das dem CHE Ranking zugrundeliegende Bewertungsschema von Forschungsleistungen ist nämlich nicht anreizkompatibel, d.h. es belohnt nicht diejenigen Forschungsleistungen, die von der Hochschulpolitik üblicherweise angestrebt werden. Die CHE Evaluatoren gleichen dem in der Kontrakttheorie gern angeführten Kolonialbeamten, der die herrschende Klapperschlangenplage bekämpfen wollte, indem er für jede Klapper einen Geldbetrag aussetzte. Ebenso wie der "Schwanzpreis" eine Verschlimmerung der Schlangenplage nach sich zog -- die Einheimischen fingen nämlich an, Klapperschlangen zu züchten -- würde die Anwendung des CHE Bewertungsschemas auf Kosten der hochwertigen Forschung eine Proliferation minderwertiger Forschungsleistungen nach sich ziehen. 
Das CHE Forschungsranking ist aber mehr als bloß ein universitätsinternes Ärgernis. Es ist geeignet, oberflächliche Betrachter der deutschen Forschungslandschaft in die Irre zu leiten und etablierten hochwertigen Forschungsstandorten Schaden zuzufügen. Kurz, das von Herrn Prof. Detlef Müller-Böling geleitete Team hat eine dilettantisch konzipierte und hochschulpolitisch gefährliche Studie vorgelegt, die in dieser Form zurückgewiesen werden muß.

Im folgenden Abschnitt stelle ich das CHE Forschungsranking kurz vor und erkläre, warum die verwendete Methode nicht adäquat ist. Daraufhin präsentiere ich im zweiten Abschnitt vier europaweit angelegte Forschungsrankings im Bereich der VWL, die den neuesten Stand der Rankingmethodologie aufzeigen. Obwohl diese vier Studien alle noch vor der Publikation des CHE Forschungsrankings im Internet veröffentlicht wurden, sind sie von den „professionellen“ CHE Evaluatoren offenbar nicht rezipiert worden. Im dritten Abschnitt vergleiche ich das CHE Forschungsranking mit den vorgestellten "top of the line" Rankings und mit dem eingangs erwähnten Ranking aus dem Jahr 1998. Der letzte Abschnitt enthält einige Anregungen zuhanden der CHE Evaluatoren. Im Anhang wurden speziell für die Leser aus Österreich und der Schweiz einige „top-20“ Ranglisten für den gesamten „deutschsprachigen“ Raum zusammengefasst.

\section{Das CHE Forschungsranking für das Fach VWL}

Die Forschungskraft der volkswirtschaftlichen Fachbereiche wird vom CHE anhand von drei objektiven Indikatoren gemessen: (1) verausgabte Drittmittel, (2) Umfang der Publikationen, und (3) Anzahl der Promotionen. Diese Evaluation beschäftigt sich mit dem zweiten objektiven Indikator, dem Ranking nach dem Umfang der Publikationen. Der Grund für die Beschränkung auf den bibliometrischen Indikator liegt nicht darin, dass an den anderen beiden Indikatoren nichts auszusetzen wäre. Beim Indikator “Anzahl der Promotionen” muss 
man in der Tat ganz ähnliche Bedenken anmelden wie beim hier untersuchten Indikator "Umfang der Publikationen" (siehe dazu Fabel et al., 2002) und der Indikator "Verausgabte Drittmittel" ist u.a. deswegen bedenklich, weil hier eine wichtige Gruppe von Interessenten, nämlich die Drittmittelgeber, den Input zur Erstellung des Rankings liefern müssen. Ich beschränke mich auf den bibliometrischen Indikator, weil in diesem Bereich Vergleiche mit anderen Rankings angestellt werden können.

Dem CHE Ranking (nach dem Umfang der Publikationen) liegt der dreijährige Untersuchungszeitraum 1998-2000 zugrunde. Erfasst werden alle Publikationen der am Stichtag an einem Fachbereich tätigen Professoren und alle deutschen Fachbereiche mit mehr als drei VWL-Professoren. Es handelt sich hierbei also um eine sogenannte «current potential» Erhebung, d.h. Forschungsleistungen werden denjenigen Fachbereichen zugeschrieben, an denen die jeweils beteiligten Forscher am Stichtag der Erhebung tätig waren. Bei dem sehr kurz gewählten Zeitraum von drei Jahren dürften sich aber die Ergebnisse nicht stark von einer sogenannten «work done at» Erhebung unterscheiden, bei der Forschungsleistungen denjenigen Fachbereichen zugeschrieben werden, denen die jeweils beteiligten Forscher im Zeitpunkt der Publikation angehörten.

Abgesehen von kleineren Mängeln (der dreijährige Untersuchungszeitraum ist sehr kurz gewählt, die Forschungsstärke eines Fachbereichs wird mit der professoralen Forschung nur teilweise erfasst), leidet das Ranking vor allem daran, dass keine vernünftige Qualitätsgewichtung des Forschungsoutputs vorgenommen wird. Obwohl die Autoren im Überblick behaupten, die erfassten Publikationen nach Art und Umfang gewichtet zu haben, zeigt sich beim kritischen Lesen, dass die vorgenommene Gewichtung lediglich auf leicht zu erfassende formale Kriterien beschränkt bleibt. Die Publikationen wurden nämlich folgendermaßen gewichtet: 
a) mit der Länge des Beitrags (bis 5 Seiten 1 Punkt; 6-10 Seiten 2 Punkte; 11-20 Seiten 3 Punkte; 20-100 Seiten 4 Punkte; über 100 Seiten 7 Punkte), ${ }^{1}$

b) mit der Anzahl der Autoren (1 Autor = 1; 2 Autoren = 0.5; 3 Autoren 0.33; mehr als 3 Autoren $=0.25$,

c) reine Herausgeberschaften: 3 Punkte,

d) graue Literatur: die Hälfte des mittels (a)-(c) ermittelten Punktewertes.

Dies bedeutet, dass alle offiziellen Publikationen mit demselben Qualitätsgewicht in die Berechnung des Forschungsoutputs eingehen. Ein Beitrag zu einem Sammelband, bei dem möglicherweise keinerlei Qualitätsanforderungen gestellt worden sind, wird somit gleich gewichtet wie ein Artikel in einer renommierten internationalen Zeitschrift mit strengem Peer Review Verfahren. Besonders bedenklich ist, dass die graue Literatur überhaupt berücksichtigt wird. Zwei Diskussionspapiere, die von der Profession eventuell nie wahrgenommen werden, erhalten so dasselbe Gewicht wie eine veröffentlichte Arbeit, mit der sich der Autor der nationalen oder sogar internationalen Konkurrenz stellt. Ein Diskussionspapier, dessen Inhalt nie veröffentlicht wird, hat eben der Diskussion nicht standgehalten und keinen wissenschaftlichen Beitrag geleistet. Es gibt somit keinen Grund, so etwas in die Bemessung des Forschungsoutputs eingehen zu lassen.

Bei einer restriktiven und vernünftigen Selektion der in Rechnung gestellten Publikationen kann eine Gleichgewichtung bei gewissen Fragestellungen vertretbar sein. Wenn aber wirklich alle Publikationen undifferenziert einbezogen werden, so erhält man bei einer

\footnotetext{
${ }^{1}$ Man beachte, dass hier bloß die rohe Seitenzahl erfasst wird und nicht etwa die auf ein Standardseitenmaß umgerechnete Länge der Publikation. Obwohl die Gewichtung nach der Länge in der Rankingliteratur durchaus kontrovers diskutiert wird, ergeben sich aber gerade im Bereich der grauen Literatur -- Diskussionspapiere werden ja oft zweizeilig geschrieben -- ohne Umrechnung auf ein Standardseitenmaß besonders gravierende Verzerrungen. Hier wird somit eine Gewissenhaftigkeit vorgetäuscht, die von der CHE Studie nicht eingelöst wird.
} 
Gleichgewichtung eine Maßzahl, die mit den in der Profession üblichen

Wertschätzungsstandards nichts mehr zu tun hat. Es vermag deshalb nicht zu verwundern, dass die “top-ten" der CHE Rangliste der am Gesamtoutput gemessen forschungsstärksten Fachbereiche (CHE: Output) für den Kenner ein ganz ungewohntes Bild abgeben:

\section{CHE: Output}

1 U Bremen

2 U Mannheim

3 U Frankfurt a.M.

4 U Hamburg

5 U Freiburg

6 U Münster

7 U Köln

8 LMU München

9 U Würzburg

10 U Potsdam

\section{CHE: Produktivität}

1 U Potsdam

2 U Würzburg

3 U Bremen

4 U Kiel

5 U Mannheim

6 EU Frankfurt a.O.

7 TU Leipzig

8 U Münster

9 U Marburg

10 U Passau

Allein der volkswirtschaftliche Fachbereich der Universität Mannheim tritt auf einem

Listenplatz auf, der angemessen erscheint. Über die anderen Platzierungen schweigt des Sängers Höflichkeit. An der Absurdität der Rangliste ändert sich auch nichts, wenn die CHE Evaluatoren versuchen, die Größe der Fachbereiche in Rechnung zu stellen, indem sie den dilettantisch berechneten Gesamtoutput der Professoren durch die Anzahl der Professoren dividieren, um so zu einer durchschnittlichen Forschungsproduktivität der "Fachbereiche" zu gelangen (CHE: Produktivität).

\section{Die "top of the line" Rankings}

Alle Forschungsrankings sind natürlich mit gewissen Schwächen behaftet. Es lassen sich aber trotzdem gute, weniger gute, und eben vollständig wertlose Rankings unterscheiden. Darüber hinaus kann man feststellen, dass die nützlichen Rankings im Zeitablauf immer besser geworden sind. Mit Hilfe der besten zur Zeit verfügbaren Forschungsrankings lassen sich durchaus vernünftige Standortbestimmungen vornehmen. 
Zu nennen sind hierbei vor allem die vier Rankingstudien, die im Jahr 2000 von der European

Economic Association in Auftrag gegeben wurden, um die europäische Forschungslandschaft im Bereich der VWL zu vermessen. In der Zwischenzeit haben die vier beauftragten Forscher bzw. Forschergruppen ihre (vorläufigen) Ergebnisse vorgelegt. Es handelt sich um die Studien von Kalaitzidakis et al. (2001), Bauwens et al. (2002), Combes und Linnemer (2002) und Coupé (2002).

Alle vier Untersuchungen beschränken sich mit Bedacht auf Zeitschriftenpublikationen, die von der Econlit Datenbank erfasst worden sind. Econlit indexierte während der vergangenen 30 Jahre ungefähr 800 Zeitschriften, sodass Tom Coupé (S. 2) zurecht anmerkt: “(O)ne can claim with slight exaggeration, first, that if one is not in Econlit, one did not do academic research in economics and second, that these journals together form the economics literature." Die vier Studien verwenden also dieselbe Datenquelle, unterscheiden sich aber insbesondere in der Auswahl der den Rankings zugrunde gelegten Zeitschriften. Einige Rankings erfassen alle Econlit Zeitschriften, andere nur die renommiertesten. Darüber hinaus werden unterschiedliche Qualitätsgewichte verwendet. Bei der Qualitätsgewichtung wird jedoch immer von der Qualität einer Zeitschrift auf die Qualität der darin veröffentlichten Artikel geschlossen. Die Qualität einer Zeitschrift wiederum wird mit Hilfe der Impaktfaktoren der Zeitschriften und eventuell zusätzlich mit Hilfe von Expertenurteilen abgeschätzt.

\footnotetext{
${ }^{2}$ Der oft gehörte Vorwurf, in der Econlit Datenbank seien die deutschen Fachzeitschriften untervertreten, ist nicht gerechtfertigt. Econlit erfasst nämlich neben den im deutschsprachigen Raum erscheinenden Zeitschriften mit gutem internationalen Renommee auch eine absolut adäquate Zahl von (z.T. ehemals) deutschsprachigen Zeitschriften, die vom internationalen Publikum weniger wahrgenommen werden; es sind dies: Allgemeines Statistisches Archiv, Aussenwirtschaft, Finanzarchiv, Ifo Studien, Jahrbuch der Wirtschaft Osteuropas, Jahrbuch für Regionalwissenschaft, Jahrbücher für Nationalökonomie und Statistik, Konjunkturpolitik, Kredit und Kapital, Mitteilungen aus der Arbeitsmarkt- und Berufsforschung, Schmollers Jahrbuch - Zeitschrift für Wirtschafts- und Sozialwissenschaften, Schweizerische Nationalbank Quartalsheft, Schweizerische Zeitschrift für Volkswirtschaft und Statistik, Wirtschaft und Recht, Wirtschaftspolitische Blätter, Zeitschrift für Betriebswirtschaft, Zeitschrift für Nationalökonomie, Zeitschrift für Wirtschafts- und Sozialwissenschaften, Zeitschrift für Wirtschaftspolitik.
} 
Die Rankings von Kalaitzidakis et al. (2001), Bauwens et al. (2002) und Coupé (2002)

beruhen auf der «work done at» Methode. Die Studie von Kalaitzidakis et al. untersucht die Periode 1995-1999 und erfasst bloß die renommiertesten 30 Zeitschriften. Die erfassten

Zeitschriften werden darüber hinaus noch mit dem entsprechenden Impaktfaktor gewichtet.

Das resultierende Ranking hängt somit sehr stark von der Publikationsinzidenz in den absolut erstklassigen Zeitschriften ab. Das hier vorgestellte Ranking von Bauwens et al. für die Jahre 1991-2000 ist demgegenüber viel weniger top-lastig. ${ }^{3}$ Diese Autoren erfassen alle Econlit Zeitschriften und gewichten die Qualität mit einem Index, der die Werte 1-10 annehmen kann. Ihre Studie erfasst aber nicht alle Wissenschaftler, sondern nur Wissenschaftler, die einen exogen vorgegebenen minimalen Publikationsoutput aufweisen. Das hier vorgestellte Ranking von Coupé für die Periode 1990-2000 ergibt sich aus dem Durchschnitt von 11 Rankings, die auf verschiedenen Methodologien beruhen. Interessanterweise ändert sich durch die unterschiedliche Machart der drei Rankings nicht allzu viel: acht Fachbereiche tauchen auf allen top-ten Listen auf.

Kalaitzidakis et al. (2001)

$\begin{array}{rlll}\text { D } & \text { EUR } & \text { Welt } & \\ 1 & 20 & 67 & \text { U Bonn } \\ 2 & 26 & 79 & \text { Humboldt U } \\ 3 & 46 & 128 & \text { LMU München } \\ 4 & 55 & 150 & \text { FU Berlin } \\ 5 & 60 & 166 & \text { U Mannheim } \\ 6 & 61 & 167 & \text { U Konstanz } \\ 7 & 67 & 177 & \text { U Bielefeld } \\ 8 & 68 & 180 & \text { U Dortmund } \\ 9 & 88 & \text { n.v. } & \text { U Karlsruhe } \\ 10 & 100 & \text { n.v. } & \text { U Freiburg }\end{array}$

Bauwens et al. (2002)

$\begin{aligned} E U^{4} & \\ 20 & \text { U Bonn } \\ 33 & \text { LMU München } \\ 45 & \text { U Mannheim } \\ 64 & \text { U Kiel } \\ 67 & \text { U Konstanz } \\ 70 & \text { FU Berlin } \\ 84 & \text { U Dortmund } \\ 88 & \text { U Bielefeld } \\ 115 & \text { U Hamburg } \\ 116 & \text { Humboldt U }\end{aligned}$

\section{Coupé (2002)}

$\begin{aligned} \text { Welt } & \\ 87 & \text { U Bonn } \\ 119 & \text { U Mannheim } \\ 147 & \text { LMU München } \\ 206 & \text { Humboldt U } \\ 225 & \text { FU Berlin } \\ 257 & \text { U Konstanz } \\ 264 & \text { U Kiel } \\ 277 & \text { U Dortmund } \\ 279 & \text { U Bielefeld } \\ 349 & \text { U Freiburg }\end{aligned}$

Im Gegensatz zu diesen auf der ,work done at“ Methode beruhenden Rankings präsentieren Combes und Linnemer (Juni und Juli 2002) (CL) echte «current potential» Rankings. In

\footnotetext{
${ }^{3}$ Ich beziehe mich hier auf das "European ranking based on the full list of journals."

${ }^{4}$ Bauwens et al. (2002) erfassen die Fachbereiche in den EU Mitgliedstaaten und Norwegen. Die EURankingplätze stammen aus einer vorläufigen Version.
} 
methodologischer Hinsicht kommen die CL Rankings - insbesondere diejenigen für die

Periode 1996-2000, auf die ich mich hier beziehe - dem CHE Ranking somit am nächsten. ${ }^{6}$

Die beiden hier präsentierten top-ten Ranglisten der am Gesamtoutput gemessen

forschungsstärksten Fachbereiche erfassen alle Econlit Zeitschriften; das erste Ranking beruht auf einer Qualitätsgewichtung (Output-QG) mit einem Index, der die Werte zwischen 1 und 12 annehmen kann, das zweite Ranking geht von einer Gleichgewichtung aus (Output-GG). ${ }^{7}$

Wiederum sieht man, dass die Reihung erstaunlich wenig sensitiv auf die Art der Qualitätsgewichtung reagiert, solange die Gewichtung nicht zu extrem ausfällt. ${ }^{8}$

\section{CL: Output-QG}

$\begin{array}{rrl}D & \text { EUR } & \\ 1 & 22 & \text { U Bonn } \\ 2 & 34 & \text { LMU München } \\ 3 & 35 & \text { U Mannheim } \\ 4 & 41 & \text { Humboldt U } \\ 5 & 58 & \text { U Konstanz } \\ 6 & 64 & \text { U Kiel } \\ 7 & 65 & \text { U Frankfurt } \\ 8 & 78 & \text { U Bielefeld } \\ 9 & 88 & \text { FU Berlin } \\ 10 & 104 & \text { U Heidelberg }\end{array}$

\section{CL: Output-GG}

$$
\text { EUR }
$$$$
22
$$$$
27
$$$$
35
$$$$
37
$$$$
38
$$$$
43
$$$$
94
$$$$
111
$$$$
116
$$$$
119
$$

U Bonn
LMU München
U Kiel
U Mannheim
U Konstanz
Humboldt U
U Frankfurt
U Bielefeld
U Heidelberg
FU Berlin

\section{CL: Produktivität}

EUR

10 LMU München (34.9)

20 U Oldenburg (7)

21 U Mannheim (41.5)

41 TU Dresden (6)

44 U Konstanz (31)

55 Humboldt U (55)

$58 \quad$ U Bonn (82.3)

61 U Bielefeld (30)

67 U Tübingen (16.5)

71 U Magdeburg (15.3)

Die CL Studie enthält auch Rankings für die Produktivität von Forschungszentren. ${ }^{9}$ Die Erhebung der Anzahl der an einem Forschungsstandort tätigen Wissenschaftler (Doktoranden wurden nicht erfasst) erfolgte aufgrund von Internetrecherchen und Email Anfragen. Die Qualität der erhobenen Daten ist somit nicht über alle Zweifel erhaben. Die Anzahl der in

\footnotetext{
${ }^{5}$ Die ,World Ranking“ Liste von Kalaitzidakis et al. (2001) umfasst bloß 200 Fachbereiche. Die Fachbereiche der Universitäten Karlsruhe und Freiburg sind auf dieser Liste nicht aufgeführt, erscheinen aber auf der „European Ranking“ Liste.

${ }^{6}$ CL stellen auch Rankings für die längere Periode 1971-2000 auf.

${ }^{7}$ Ich beziehe mich hier auf die in Part II aufgeführten „Rankings of European Research Centers, 1996-2000,“ CLpn (QG) und E1n (GG).

${ }^{8}$ Combes und Linnemer präsentieren auch Rankings, die nur die acht renommiertesten Zeitschriften erfassen.

${ }^{9}$ Bauwens et al. (2002) stellen ebenfalls ein Produktivitätsranking auf (siehe Anhang); aber es wird dort die durchschnittliche Produktivität der "produktiven" Wissenschaftler gemessen, und dies ist eigentlich nicht das, was von primärem hochschulpolitischem Interesse ist. Ähnlich konzipiert sind Rankings der z.B. 10 produktivsten Wissenschaftler pro Fachbereich (cf. Coupé, 2002, Table 7). Interessanter sind die ebenfalls von CL präsentierten Produktivitätsrankings, welche die Altersstruktur der evaluierten Fachbereiche berücksichtigen.
} 
Rechnung gestellten Mitarbeiter ist in der Rangliste (CL: Produktivität) dem Namen des

Fachbereichs nachgestellt. ${ }^{10}$

\section{Ein Vergleich der Rankings}

Was die Spitzenplätze anbelangt, so stimmen die vier im vorangehenden Abschnitt vorgestellten „top of the line“ Rankings sehr gut überein. Es lassen sich deshalb recht eindeutige Schlussfolgerungen ziehen. Die unbestrittenen vier top-Standorte der volkswirtschaftlichen Forschung in Deutschland sind

1 U Bonn,

2 LMU München,

3 U Mannheim und

4 Berlin (FU und HU).

Dabei liegt Bonn klar vorn, gefolgt vom Duo Mannheim und München. Der Standort Berlin profitiert von zwei starken Fachbereichen, liegt zur Zeit aber wohl (noch) an vierter Stelle. Hinter diesem Quartett folgen zwei weitere Standorte, nämlich

5 U Konstanz und

6 U Kiel.

Die letzten beiden Standorte, die klar zur Spitzengruppe gehören sind:

$7 \quad$ U Bielefeld und

8 U Dortmund.

Die Diskrepanz zwischen diesem Resultat und der hier noch einmal präsentierten CHE

Rangliste ist frappant:

\section{CHE: Output}

1 U Bremen

2 U Mannheim

3 U Frankfurt a.M.

4 U Hamburg

5 U Freiburg

6 U Münster

7 U Köln

8 LMU München "top of the line" Rankings

1 U Bonn

2 U Mannheim

3 LMU München

4 Berlin (HU und FU)

5 U Konstanz

6 U Kiel

7 U Bielefeld

8 U Dortmund

\section{BU: Output}

1 U Bonn

2 LMU München

3 U Mannheim

4 FU Berlin

5 U Konstanz

6 U Kiel

7 U Dortmund

8 U Bielefeld

\footnotetext{
${ }^{10}$ Ich beziehe mich hier auf das in Part II aufgeführte "Ranking of European Research Centers Adjusted per Faculty Members, 1996-2000," CLpn.
} 
Vergleicht man darüber hinaus das etwas ältere Ranking von Bommer und Ursprung (1998) (BU), das sich auf den Zeitraum 1990-1995 bezog, mit der obigen Zusammenfassung der vier „top of the line“ Rankings, so fällt auf, dass sich in den letzten 5 Jahren offenbar in der deutschen Forschungslandschaft im Bereich der VWL nicht viel verändert hat.

Ein ähnliches Bild ergibt sich, wenn man die Produktivitätsrankings betrachtet. Auch hier zeigt sich eine viel größere Übereinstimmung zwischen dem „top of the line“ Ranking von CL für die Periode 1996-2000 und dem BU-Ranking für die Periode 1990-1995 als zwischen diesen beiden Rankings und dem CHE Ranking für die Periode 1998-2000. Außer der Humboldt Universität, die 1990-1995 noch in der Aufbauphase war, sind alle großen Fachbereiche der top-8 Rangliste von CL (München, Mannheim, Konstanz, Bonn und Bielefeld) auch schon auf der top-8 Rangliste von BU zu finden.

\section{CHE: Produktivität}

1 U Potsdam

2 U Würzburg

3 U Bremen

4 U Kiel

5 U Mannheim

6 EU Frankfurt a.O.

7 U Leipzig

8 U Münster

\section{CL: Produktivität}

1 LMU München (34.9)

2 U Oldenburg (7)

3 U Mannheim (41.5)

4 TU Dresden (6)

5 U Konstanz (31)

6 Humboldt U (55)

7 U Bonn (82.3)

8 U Bielefeld (30)

\section{BU: Produktivität}

1 U Bonn

2 U Dortmund

3 LMU München

4 U Mannheim

5 U Konstanz

6 U Bielefeld

7 U Kiel

8 U Osnabrück

Eine große Schwachstelle bei den Produktivitätsberechnungen liegt bei der korrekten und gleichartigen Erfassung der Anzahl der in Rechnung gestellten Wissenschaftler. Insbesondere bei den kleineren hier aufgeführten Fachbereichen erscheint die von CL veranschlagte Größenordnung etwas suspekt. Darüber hinaus fällt auf, dass der Fachbereich der Universität Dortmund in der CL Studie gar nicht in Erscheinung tritt. Worauf dies zurückzuführen ist, konnte bisher nicht geklärt werden. Diese Ungereimtheiten sind bei den zu ziehenden Schlussfolgerungen zu berücksichtigen. 


\section{Die Zukunft institutioneller Evaluatoren}

Man ist vielleicht versucht, aus diesen Ausführungen den Schluß zu ziehen, dass in Zukunft Forschungsrankings nicht von institutionellen Evaluatoren bereitgestellt werden sollten. Eine derartige Schlußfolgerung wäre aber übereilt, denn die routinemäßige Erstellung von Rankings ist ebenso wenig eine wissenschaftliche Fragestellung wie die maßgeschneiderte Aufbereitung des Materials für die verschiedenen Interessenten. Die Absicht der Hochschulrektorenkonferenz, mit dem CHE eine Institution zu schaffen, die den Leitungsorganen der Universitäten zentral bereitgestellte Führungsinstrumente anbietet, ist deshalb durchaus vernünftig gewesen. Was bisher jedoch gefehlt hat, sind erstens klar formulierte Anforderungsprofile bezüglich der gewünschten Produkte und zweitens eine Qualitätsüberwachung, die in einem nichtkompetitiven Umfeld unabdingbar ist. Es sind eben nicht bloß die Universitäten, die in Ermangelung von Marktkräften gemäß CHE Homepage (http://www.che.de) ,wachgeküsst“" werden müssen.

Eines dürfte jedoch klar geworden sein: die Stärke von institutionellen Evaluatoren liegt nicht in der Erarbeitung innovativer Methoden. Hier hat die Wissenschaft einen klaren Vorteil. Das CHE sollte deshalb in Zukunft die Hände von „originellen“ Eigenkreationen lassen. Der Beitrag institutioneller Evaluatoren muß darin bestehen, die von der Wissenschaft bereitgestellten Methoden aufzunehmen, um damit dem Auftrag zur Bereitstellung von Führungsinstrumenten nachzukommen. Im vorliegenden Fall bedeutet dies konkret, dass das CHE die vorliegenden vier „top of the line“ Rankings eingehend analysieren sollte, um (1) die sicherlich vorhandenen Datenmängel im Bereich der deutschen Fachbereiche zu beheben, (2) die Abgrenzung der Forschungseinheiten (Fachbereiche, Forschungszentren, etc.) und Disziplinen (VWL, BWL) so vorzunehmen, wie es die jeweilige Fragestellung erfordert, (3) die Information so aufzubereiten, dass die Führungsorgane der Universitäten sie sinnvoll 
nutzen können. Eventuell könnten institutionelle Evaluatoren auch zusätzliche Daten erheben, mit denen sich gewisse Fragestellungen direkter und präziser beantworten lassen. ${ }^{11}$

Diese Evaluation eines Evaluators ist somit als ein Plädoyer für eine bessere Zusammenarbeit zwischen Wissenschaft und Evaluationsinstitutionen zu verstehen; ein Kuss eben -- und nicht etwa ein Fußtritt.

\footnotetext{
${ }^{11}$ Wenn man z.B. die Altersstruktur bei Forschungsrankings berücksichtigen will, so wie dies Combes und Linnemer (2002) tun, sind präzise Angaben über das Lebensalter bzw. den Zeitpunkt des Ausbildungsabschlusses der Wissenschaftler hilfreich.
} 


\title{
Literaturverzeichnis
}

Bauwens, L., A. Kirman, M. Lubrano und C. Protopopescu: Ranking European economic departments: A statistical approach, July 2002. http://durandal.cnrs-mrs.fr/GREQAM/cv/kirman.htm

Berghoff, S., G. Federkeil, P. Giebisch, C.-D. Hachmeister und D. Müller-Böling: Das Forschungsranking deutscher Universitäten, Arbeitspapier Nr. 40, Centrum für Hochschulentwicklung, November 2002. http://www.che.de/html/forschungsranking.htm

Bommer, R. und H. Ursprung: Spieglein, Spieglein and der Wand: Eine publikationsanalytische Erfassung der Forschungsleistungen volkswirtschaftlicher Fachbereiche in Deutschalnd, Österreich und der Schweiz, Zeitschrift für Wirtschaftsund Sozialwissenschaften 118, 1998, 1-28.

Combes, P. und L. Linnemer: Measuring and ranking economics throughout Europe, June 2002. http://www.crest.fr/pageperso/lei/linnemer/linnemer.htm

Combes, P. und L. Linnemer: Measuring and ranking economics throughout Europe: Part II of a report to the EEA, July 2002.

http://www.crest.fr/pageperso/lei/linnemer/linnemer.htm

Coupé, T.: Revealed performances: Worldwide rankings of economists and economics departments, 2002.

http://homepages.ulb.ac.be/ tcoupe/updaterevealedperformances.pdf

Fabel, O., E. Lehmann und S. Warning: Vorträge als Qualitätsindikator: Empirische Evidenz der Jahrestagungen des Vereins für Socialpolitik, Diskussionspapier Serie I - Nr. 321, Fachbereich Wirtschaftswissenschaften, Universität Konstanz, November 2002.

Kalaitzidakis, P., T. Mamuneas und T. Stengos: Rankings of academic journals and institutions in economics, October 2001.

http://www.econ.ucy.ac.cy/papers/0110.pdf

\begin{abstract}
This is a comment on a ranking of German economics departments published by a semiofficial institution, the Center for Higher Education Development (CHE). It is shown that the CHE ranking is highly misleading because the publication output is not weighted according to quality. The CHE ranking is compared to the results of four recent ranking studies commissioned by the European Economic Association (EEA) and an earlier ranking of German, Austrian and Swiss economics departments. For the readers from Austria and Switzerland we include in the appendix a "peek preview" of the "EEA rankings" which includes the top-ranked Austrian and Swiss departments.
\end{abstract}




\section{Anhang}

Top-20 Rankings des gesamten Forschungsoutputs ${ }^{1}$

$\begin{array}{llllll}\text { CHE: Output } & \text { Kalaitzidakis et al. } & \text { Bauwens et al. } & \text { CL: Output-QG } & \text { Coupé } & \text { BU: Output } \\ \begin{array}{l}\text { Bremen } \\ \text { Mannheim }\end{array} & \text { U Wien } & \text { Bonn } & \text { Bonn } & \text { Bonn } & \text { Bonn } \\ \text { Frankfurt a.M. } & \text { Humboldt U } & \text { LMU München } & \text { LMU München } & \text { Mannheim } & \text { LMU München } \\ \text { Hamburg } & \text { Genf } & \text { Mannheim } & \text { Mannheim } & \text { U Wien } & \text { Mannheim } \\ \text { Freiburg i.B. } & \text { Zürich } & \text { Kiel } & \text { Humboldt U } & \text { GMU München } & \underline{\text { U Wien }} \\ \text { Münster } & \text { LMU München } & \text { Konstanz } & \text { U Wien } & \text { Zürich } & \text { Basel } \\ \text { Köln } & \text { FU Berlin } & \text { FU Berlin } & \text { Konstanz } & \text { Humboldt U } & \text { Zürich } \\ \text { LMU München } & \text { Mannheim } & \text { Linz } & \text { St.Gallen } & \text { FU Berlin } & \text { TU Wien } \\ \text { Würzburg } & \text { Konstanz } & \text { Dortmund } & \text { Kiel } & \text { Basel } & \text { FU Berlin } \\ \text { Potsdam } & \text { Lausanne } & \text { Bielefeld } & \text { Frankfurt a.M. } & \text { Konstanz } & \text { Konstanz } \\ \text { Heidelberg } & \text { Bielefeld } & \text { TU Wien } & \text { Bielefeld } & \text { Kiel } & \underline{\text { Linz }} \\ \text { Humboldt U } & \text { Dortmund } & \text { Hamburg } & \text { FU Berlin } & \text { Dortmund } & \text { Lausanne } \\ \text { EU Frankfurt a.O. } & \text { Basel } & \text { Humboldt U } & \text { Heidelberg } & \text { Bielefeld } & \text { Kiel } \\ \text { Leipzig } & \text { St.Gallen } & \text { Osnabrück } & \text { Linz } & \text { TU Wien } & \text { Dortmund } \\ \text { Marburg } & \text { Karlsruhe } & \text { Regensburg } & \text { Hamburg } & \text { Lausanne } & \text { Bielefeld } \\ \text { FU Berlin } & \text { TU Wien } & \text { Augsburg } & \text { HEI Genf } & \text { Linz } & \text { Bern } \\ \text { Kiel } & \text { Freiburg i.B. } & \text { Karlsruhe } & \text { EU Frankfurt a.O. } & \text { Bern } & \text { Saarbrücken } \\ \text { Oldenburg } & \text { Linz } & \text { Oldenburg } & \text { Tübingen } & \text { St.Gallen } & \text { Regensburg } \\ \text { Mainz } & \text { Saarbrücken } & \text { Tübingen } & \text { Bern } & \text { Freiburg } & \text { Tübingen } \\ \text { Trier } & \text { Bern } & \text { Freiburg i.B. } & \text { U Hohenheim } & \text { Karlsruhe } & \text { Hannover }\end{array}$

\footnotetext{
${ }_{1}^{1}$ Die entsprechenden Literaturhinweise finden sich im Text.

${ }^{2}$ Man beachte, dass in Bauwens et al. (2002) die Fachbereiche in der Schweiz nicht erfasst werden.
} 


\section{Top-20 Rankings der Forschungsproduktivität}

\section{CH : Produktivität CL: Produktivität BU: Produktivität BKLP: Produktivität ${ }^{3}$}

\begin{tabular}{|c|c|c|c|}
\hline Potsdam & LMU München & Basel & $\underline{\operatorname{Linz}}(13)$ \\
\hline Würzburg & Oldenburg & U Wien & $\overline{\text { TU Wien }(10)}$ \\
\hline Bremen & Mannheim & TU Wien & Oldenburg (5) \\
\hline Kiel & HEI Genf & $\overline{\text { Bonn }}$ & LMU München (33) \\
\hline Mannheim & TU Dresden & Dortmund & FU Berlin (20) \\
\hline EU Frankfurt a.O. & Konstanz & $\underline{\operatorname{Linz}}$ & Karlsruhe (7) \\
\hline Leipzig & $\underline{\text { U Wien }}$ & LMU München & $\underline{\text { U Wien (37) }}$ \\
\hline Münster & $\overline{\text { Humboldt U }}$ & Mannheim & Dortmund (18) \\
\hline Marburg & Bonn & Zürich & Bamberg (4) \\
\hline Passau & Bielefeld & Konstanz & Bonn (57) \\
\hline Oldenburg & Tübingen & Genf & Osnabrück (10) \\
\hline Bayreuth & Magdeburg & Bielfeld & Mannheim (38) \\
\hline Köln & Lausanne & Kiel & Heidelberg (5) \\
\hline Freiburg i.B. & Zürich & Osnabrück & Essen (6) \\
\hline TU Berlin & Heidelberg & Bern & U Graz (3) \\
\hline Humboldt U & Essen & FU Hagen & TU Graz (1) \\
\hline LMU München & Frankfurt a.M. & Regensburg & $\overline{\text { Konstanz }}(26)$ \\
\hline Osnabrück & $\underline{\operatorname{Linz}}$ & Augsburg & Hamburg (15) \\
\hline Jena & $\overline{\mathrm{DU}}$ Krems & ETH Zürich & Hannover (6) \\
\hline Hamburg & $\overline{\text { Mainz }}$ & Saarbrücken & Bielefeld (21) \\
\hline
\end{tabular}

\footnotetext{
${ }^{3}$ Die Maßzahl "average score" von Bauwens et al. (2002) ist keine echte Produktivität, weil nur Wissenschaftler mit einem vorgegebenen minimalen Forschungsoutput erfasst werden. Die Anzahl der im jeweiligen Fachbereich so erfassten Wissenschaftler ist dem Namen des Fachbereichs nachgestellt. Man beachte wiederum, dass die Studie von Bauwens et al. die Fachbereiche in der Schweiz nicht erfasst. Die LMU München wurde in der vorläufigen Version, aus der diese Rangliste entnommen wurde, ungenügend erfasst und fehlt deshalb.
} 